\title{
An interventional pilot of customized adherence enhancement combined with long- acting injectable antipsychotic medication (CAE-L) for poorly adherent patients with chronic psychotic disorder in Tanzania
}

\author{
Jessie Mbwambo ${ }^{1}$, Sylvia Kaaya ${ }^{1}$, Isaac Lema' ${ }^{1}$ Christopher J. Burant ${ }^{2}$, Catherine Magwiza' , Kim Madundo ${ }^{1}$,
} Godwin Njiro ${ }^{1}$, Carol E. Blixen ${ }^{3}$, Kristin A. Cassidy ${ }^{3}$, Jennifer B. Levin ${ }^{3}$ and Martha Sajatovic ${ }^{4 *}$

\begin{abstract}
Background: Chronic psychotic disorders (CPD) impose a particularly significant burden in resource-limited settings. Combining long-acting antipsychotic medication (LAl) with a customized adherence enhancement intervention (CAE-L) has potential to advance care.

Methods: Nineteen adults $\geq$ age 18 with CPD who self-reported missing $\geq 20 \%$ of antipsychotic medication within the last month were stabilized on oral haloperidol prior to transitioning to monthly haloperidol decanote for 25 weeks. Outcome evaluations were conducted at baseline and Week 25. Primary outcomes were oral medication adherence assessed via the Tablet Routines Questionnaire (TRQ) and LAI injection frequency. Secondary outcomes included CPD symptoms measured by the Brief Psychiatric Rating Scale and Clinical Global Impressions, functioning evaluated using the Social and Occupational Functioning Scale, and medication attitudes assessed with the Drug Attitudes Inventory.

Results: Mean sample age was $38.79(\mathrm{SD}=9.31)$ with 18 individuals completing the study. There was one serious adverse event, a relapse into substance use, not deemed study-related. Mean endpoint LAl dosage was $65.79 \mathrm{mg}$ $(S D=22.38)$. TRQ mean scores were $21.84(S D=13.83)$ and $12.94(S D=11.93)$ at screen and baseline respectively. For only two individuals who were on concomitant oral medication at 25 weeks, TRQ change was not calculated. LAl injection frequency was $100 \%$. Medication attitudes scores significantly improved from $7.89(S D=2.72)$ to 9.83 $(\mathrm{SD}=0.52)(p=.001$.) Changes in CPD symptoms and functioning were non-significant.
\end{abstract}

Conclusions: CAE-L appears to be preliminarily feasible and acceptable in Tanzanians with CPD.

Trial registration: The study was registered on ClinicalTrials.gov (NCT04327843) on March 31, 2020.

Keywords: Schizophrenia, Treatment adherence, Antipsychotic, Psychosis

*Correspondence: martha.sajatovic@uhhospitals.org

${ }^{4}$ Department of Psychiatry \& of Neurology, Case Western Reserve University School of Medicine and University Hospitals Cleveland Medical Center, W.O. Walker Bldg, 7th Floor, 10524 Euclid Avenue, Cleveland, OH 44106, USA

Full list of author information is available at the end of the article

\section{Introduction}

Chronic psychotic disorders (CPDs) occur world-wide but impose a particularly significant burden in resourcelimited settings where the professional workforce is spread thin and access to both medication and behavioral treatments may be limited [1]. In Sub-Saharan Africa

(c) The Author(s) 2022. Open Access This article is licensed under a Creative Commons Attribution 4.0 International License, which permits use, sharing, adaptation, distribution and reproduction in any medium or format, as long as you give appropriate credit to the original author(s) and the source, provide a link to the Creative Commons licence, and indicate if changes were made. The images or other third party material in this article are included in the article's Creative Commons licence, unless indicated otherwise in a credit line to the material. If material is not included in the article's Creative Commons licence and your intended use is not permitted by statutory regulation or exceeds the permitted use, you will need to obtain permission directly from the copyright holder. To view a copy of this licence, visit http://creativecommons.org/licenses/by/4.0/. The Creative Commons Public Domain Dedication waiver (http://creativeco mmons.org/publicdomain/zero/1.0/) applies to the data made available in this article, unless otherwise stated in a credit line to the data. 
(SSA), as is the case for people with CPD in other parts of the world, poor medication adherence is seen in approximately half of individuals with CPD and is a major driver of relapse [2-6].

Reflecting the broad use of antipsychotic medications, a recent report from Namibia found that antipsychotic medications were the most widely consumed psychotropic medicines (84\% were anti-psychotics) vs. $9.2 \%$ anti depressants and $6.8 \%$ anxiolytics [7]. Because a major impediment to adherence in CPD is difficulty with consistent medication routines, long-acting injectable antipsychotic medication (LAI) can be a potentially efficient and effective treatment option [8,9]. An advantage for LAI is that can be administered monthly or even less frequently, eliminating the daily need to take medications which in itself can be a stigmatizing behavior [10]. But medication alone is unlikely to modify long-term attitudes and behaviors, and LAI is not a stand-alone care approach for CPD given the long-term and individual care needs of people with CPD $[11,12]$.

A brief, practical behavioral approach that maps onto individual patient reasons for poor adherence and which is intended to be used as a complement to LAI has been developed by a U.S. study team $[13,14]$. To be feasible in lower-resource settings, effective interventions need to be able to be delivered by diverse types of staff, should be evidence-based, and should be able to be readily scaled-up.

Combining LAI with a customized adherence enhancement behavioral intervention (CAE-L) is an approach that has potential to advance care for people with $\mathrm{CPD}$ in resource-limited settings. This report describes a firstever testing of CAE-L in poorly adherent patients with CPD in Tanzania.

\section{Materials and methods}

\section{Overview}

This as a 6-month prospective, non-controlled trial of CAE-L in 20 poorly adherent patients with CPD in Dar es Salaam, Tanzania. The study is part of a larger U.S. National Institute of Mental Health (NIMH)-funded trial described in greater detail elsewhere [15]. The LAI used in the study, haloperidol decanote, is widely available for the treatment of individuals with CPD in SSA $[7,16]$. The behavioral intervention used in the study (Customized Adherence Enhancement/CAE) was delivered by social workers who were trained to follow a detailed curriculum. The study team used a mix of qualitative and quantitative evaluations to adapt the U.S. version of CAE to be culturally and linguistically appropriate for this setting [15]. Patient outcome assessments included adherence behaviors and attitudes, CPD symptoms, and functional status.

\section{The CAE-L intervention}

The CAE-L intervention was previously tested in 2 U.S. studies involving patients with CPD. Patients received monthly CAE combined with LAI (CAE-L) for 6 months $[14,17]$. In one of the U.S. studies, the LAI used was haloperidol decanoate, a first-generation LAI that is widely available in lower-resources settings [16, 17]. Drawn from iterative pilot work, CAE-L is flexibly delivered as a series of up to 4 treatment behavioral modules for which use is determined based upon an individual's reasons for non-adherence (adherence barriers) [10, 14, 18, 19]. Adherence barriers are identified using 2 standardized measures, the Rating of Medication Influences (ROMI) and the Attitudes toward Mood Stabilizers Questionnaire (AMSQ) [20-22]. The 4 available modules are: 1) Psychoeducation focused on medication and consequences of missing medication; 2) Modified Motivational Enhancement Therapy (MET) to address non-adherence related to substance use; 3) Communication with Providers to facilitate appropriate treatment expectations and optimize management of feared or experienced side effects; 4) and Medication Routines intended to incorporate medication-taking into lifestyle. The modules are intended to be delivered by a clinical staff member during the same visit that the individual with CPD receives their LAI injection.

\section{Intervention site}

The study setting where patients were enrolled was Muhimbili National Hospital, a 70-bed national referral hospital located in urban Dar es Salaam, Tanzania. It is the only psychiatric national referral center and serves a population of approximately 4.5 million. Patients are referred from 4 catchment zones that include 3 regional public and private hospitals.

\section{Study population}

The sample comprised 19 adult patients $\geq$ age 18 with schizophrenia who self-reported missing $20 \%$ or more of antipsychotic medication within the last month, an established benchmark for poor adherence [23]. Adherence was measured using the Tablets Routines Questionnaire (TRQ) which measures medication adherence as a percentage of medication missed or skipped [24, 25]. Eligible patients had to agree to receive LAI and be able to participate in research activities. Written informed consent was obtained from all subjects/patients. Exclusion Criteria will include: 1 .) Individuals on LAI immediately prior to enrollment, or those with intolerance or resistance to LAI; 2.) Medical conditions that would interfere with the patient's ability to participate in the trial; 3.) Physical dependence on substances likely to lead to withdrawal 
reaction; 4.) Immediate risk of harm to self or others; and 5.) Pregnancy or lactation.

\section{LAI}

Patients already on oral haloperidol were switched to haloperidol decanoate and stabilized. Individuals not on antipsychotic medication at the time of screening assessment or those on a different antipsychotic medication received an oral tolerance test (OTT) consisting of up to 14 days of oral haloperidol $4-10 \mathrm{mg} /$ day. If the OTT suggested good tolerability, the participant then received LAI (haloperidol decanoate) intramuscularly (IM) after completion of baseline assessments. Dosing of LAI, given once every month for 6 months, was prescribed as clinically indicated by the treating research psychiatrist using conservative dosing to minimize drug-related adverse effects.

\section{Concomitant treatments}

Stable dose oral psychotropic drugs ( $>30$ days of previous use) other than antipsychotics were continued. New psychotropic medication starts were not permitted. Medications for side effects could be given at the discretion of the treating research psychiatrist.

\section{Study measures}

Baseline information included duration of psychiatric illness, past hospitalizations, past antipsychotic medication treatment history, and cumulative medical burden as evaluated by the self-reported Charlson Index [26]. Substance use was measured with the Alcohol Use Disorders Identification Test (AUDIT) and the Alcohol, Smoking and Substance Involvement Screening Test (ASSIST) [27, 28].

Outcome assessments were conducted at study baseline and at Week 25 follow-up (study endpoint). Similar to U.S. studies testing this blended LAI + behavioral intervention $[13,14]$ the primary outcomes were change in oral psychotropic medication adherence (for those who were also on oral drug at both study baseline and study endpoint) as measured by the TRQ and the mean LAI injection frequency. A participant was considered adherent if LAI was administered within 1 week of the time that it was originally scheduled to be administered. Secondary outcomes were change in adherence attitudes as measured by the 10-item Drug Attitude Inventory (DAI) and change in CPD symptoms as measured by the 18-item Brief Psychiatric Rating Scale (BPRS) and the single item Clinical Global Impressions (CGI) [2931]. The BPRS version scoring ranged from 0 (no symptoms) to 7 (most severe). Change in functional status was evaluated using the Social and Occupational Functioning Scale (SOFAS) [32] .

\section{Safety/laboratory evaluations}

Safety evaluations included basic laboratory evaluations (serum comprehensive metabolic panel, lipid profile, $\mathrm{CBC}$ with differential, and HIV as well as urine pregnancy testing for women) and EKG. Patient vital signs and weight were collected at each study visit. Standardized measures of extrapyramidal symptoms were assessed with Extrapyramidal Symptoms Scale-Abbreviated version (ESRS-A) [33]. Finally, reported side effects were evaluated at each study visit using a standardized format.

\section{Data analysis}

As this study was mainly focused on feasibility, patient acceptability, and research capacity-building, we assessed only descriptive statistics and change from baseline in the primary and secondary measures using dependent samples paired t-tests to assess pre-post data in the sample that completed the study. This parametric technique was used, as there were no violations in the assumptions for the analyses (e.g., normality in the mean difference scores) and the techniques was robust to type 2 error. Data analysis was conducted using SPSS ver. 27 software.

\section{Results}

\section{Study enrollment}

Figure 1 illustrates study flow and enrollment. Screening evaluations began on Oct 28, 2019 and the first participant was enrolled on Nov 5, 2019. There were 38 individuals who were initially approached for enrollment, with 22 individuals who appeared to fit preliminary study entry criteria and provided informed consent. Of these, 2 individuals were unable to tolerate OTT and did not proceed to study baseline. There were 19 individuals who completed baseline evaluations. Of these, all individuals participated in all study procedures and assessments except for one individual who missed the endpoint study assessment at week 25 . All study participants were outpatients.

\section{Baseline sample}

Table 1 shows the baseline study sample. Notably, this was a relatively young sample with a mean age of 38.79 $(\mathrm{SD}=9.31), 73.7 \%$ male $(n=14)$ with a CPD duration of $18.89(\mathrm{SD}=10.87)$ years. Most had multiple previous hospitalizations for CPD and had been treated with a variety of oral antipsychotic medications in the past. None had been on haloperidol decanoate in the past. As this was a clinically "stable" sample at baseline, mean total BPRS and CGI scores were relatively low. 


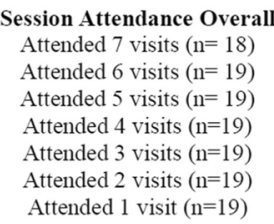

Fig. 1 Study flow and enrollment

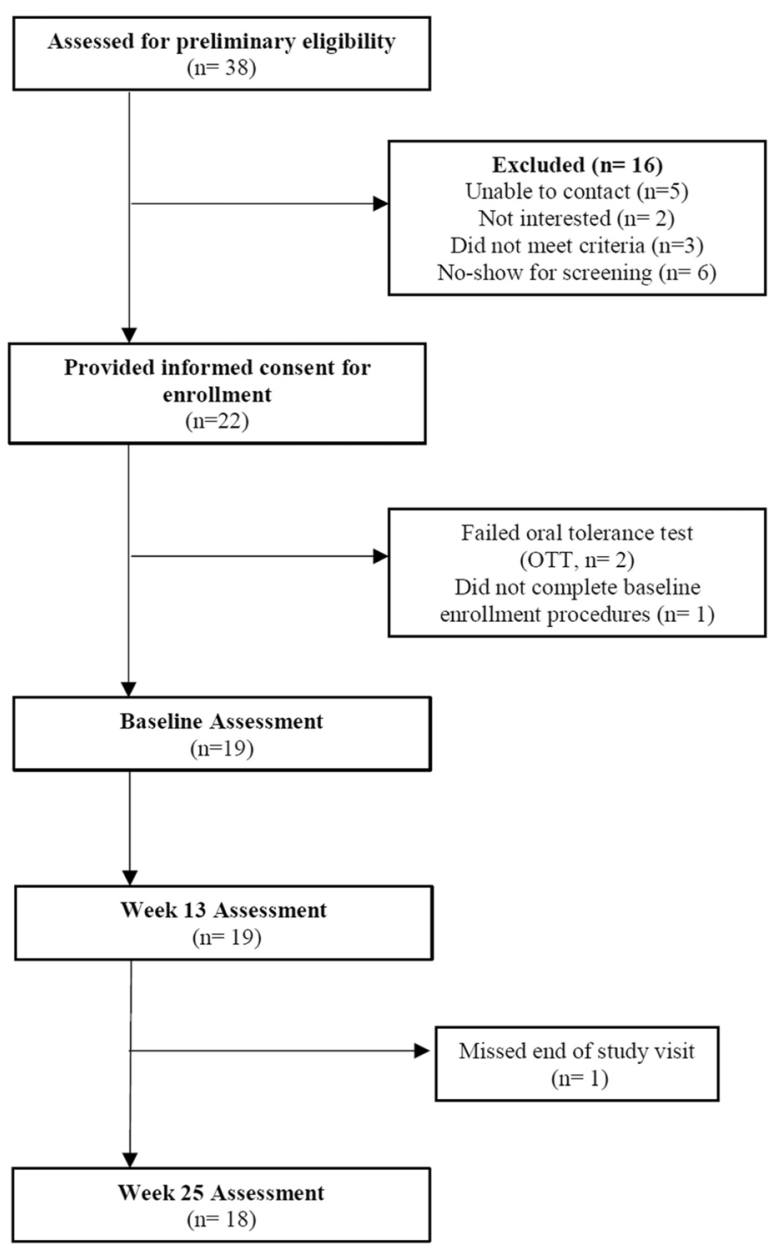

\section{Safety data and LAI dosage}

Among the 19 individuals who completed baseline evaluation and went on to received CAE-L, there was 1 serious adverse event (SAE) in which 1 individual had a relapse into substance use and was breifly hospitalized. This SAE was not deemed to be related to this individual's particiaption in the study. In addition, 13 participants had reported side effects at some point during the 25 week trial. All reported side effects were mild. There were only 2 participants who reported side effects present during the last visit. One individual reported blurry vision during the last visit which was not reported in the previous visits. Another individual reported mild muscle pain during that was present at baseline, resolved during most of the course of the trial, and then was reported again at study endpoint. Out of the 19 individuals who received medication treatment, the most common side effects were tremor $(N=7,37 \%)$, drowsiness $(N=6.32 \%)$ blurry vision $(N=3,16 \%)$, restlessness $(N=2,11 \%)$, and muscle pain $(\mathrm{N}=2,11 \%)$. The following were reported once: dry mouth, bad taste, nausea, twitching, headache, dry eyes and lightheadedness. No side effects were associated with study discontinuation. The mean endpoint dosage of haloperidol decanoate was $65.79 \mathrm{mg}(\mathrm{SD}=22.38)$. There were no EKG or laboratory testing changes that were deemed to be clinically significant.

\section{CPD and other health outcomes}

Table 2 shows group mean scores for the key outcomes of interest. Mean TRQ score, calculated only for oral medications, was $21.84(\mathrm{SD}=13.83)$ at screening. TRQ scores at baseline could only be calculated for 18 individuals and showed a mean of $12.94(\mathrm{SD}=11.93)$. As only 2 individuals were on concommitten oral CPD mediation at 25 week follow up, mean change in TRQ was not calculated. LAI injection frequency was 100\%. Mean baseline scores on BPRS and CGI decreased from 27.00 $(\mathrm{SD}=10.26)$ to $25.06(\mathrm{SD}=8.53)$ and $2.88(\mathrm{SD}=1.32)$ to $2.24(\mathrm{SD}=1.09)$ respectively at the 25 -week followup, a non-significant change. Mean SOFAS score change 
Table 1 Baseline demographic information of enrolled participants $(N=19)$

\begin{tabular}{|c|c|c|}
\hline Variable & Mean (SD) & $\mathrm{N}(\%)$ \\
\hline Age in years & 38.79 (9.31) range (27-64) & \\
\hline \multicolumn{3}{|l|}{ Sex } \\
\hline Male & & $14(73.7 \%)$ \\
\hline Female & & $5(26.3 \%)$ \\
\hline \multicolumn{3}{|l|}{ Marital status } \\
\hline Single/never married & & $12(63.2 \%)$ \\
\hline Married & & $2(10.5 \%)$ \\
\hline Separated/divorced & & $4(21.1 \%)$ \\
\hline Widowed & & $1(5.3 \%)$ \\
\hline Educational level in years & 8.37 (2.99) range (5-17) & \\
\hline \multicolumn{3}{|l|}{ Employment } \\
\hline Full-time & & $4(21.1 \%)$ \\
\hline Part-time & & $3(15.8 \%)$ \\
\hline Unemployed & & $12(63.2 \%)$ \\
\hline Age of CPD onset in years & 19.89 (4.47) range (15-33) & \\
\hline Duration of CPD in years & 18.89 (10.87) range (4-47) & \\
\hline \multicolumn{3}{|l|}{ Number of previous hospitalizations } \\
\hline For CPD & $6.16(7.55)$ range $(0-30)$ & \\
\hline For substance abuse & $0.05(.23)$ range $(0-1)$ & \\
\hline Past physical abuse & & $6(31.6 \%)$ \\
\hline Past sexual abuse & & $1(5.3 \%)$ \\
\hline Family history mental illness & & $10(52.6 \%)$ \\
\hline Family history substance abuse & & $10(52.6 \%)$ \\
\hline AUDIT score & 1.84 (3.13) range (0-12) & \\
\hline ASSIST score & $1.58(2.65)$ range $(0-10)$ & \\
\hline Self-reported Charlson Index Score & $0.37(0.50)$ range $(0-1)$ & \\
\hline Body Mass Index (BMI) & $22.70(4.89)$ range (15.62-34.29) & \\
\hline \multicolumn{3}{|l|}{ Past oral medication history } \\
\hline Chlorpromazine & & $8(42.1 \%)$ \\
\hline Fluphenizine & & $11(57.9 \%)$ \\
\hline Haloperidol & & $14(73.7 \%)$ \\
\hline Olanzapine & & $3(15.8 \%)$ \\
\hline Promethazine & & $8(42.1 \%)$ \\
\hline Risperidone & & $5(26.3 \%)$ \\
\hline Screening $\mathrm{TRQ}^{\mathrm{a}}$ & $21.84(\mathrm{SD}=13.83)$ & \\
\hline Baseline TRQ $^{\mathrm{a}}$ & $12.94(11.93)$ range $(0-33)$ & \\
\hline
\end{tabular}

a Tablets Routine Questionnaire: self-reported proportion of missed oral medication in the last 30 days. Scores range from "0" (perfect adherence) to "100" (did not take any prescribed medication). Mean Tablet Routines Questionnaire calculated only for oral CPD medications (antipsychotics, mood stablizers, antidepressants). If more than 1 oral medication was prescribed. an average TRQ was calculated. Screening sample TRQ, $N=19$, Baseline sample TRQ, $N=18$

$C P D$ chronic psychotic disorders

AUDIT Alcohol Use Disorders Identification Test

ASSIST Alcohol, Smoking, and Substance Involvement Screening Test

was also non-significant. In contrast to their being no change in symptoms, total DAI score improved from 7.89 $(\mathrm{SD}=2.72)$ at baseline to $9.83(\mathrm{SD}=0.52)$, a significant improvement $(p=.001)$.

ESRS scores were relatively unchanged from 0.06 at baseline and 0.06 at endpoint for Parkinsonism; 0.00 to 0.00 for Dystonia; 0.17 to 0.11 ; and 0.28 to 0.00 for Akathasia. BMI was also relatively unchanged. In addition to standardized measures of CPD and other health outcomes, we also assessed patient-perceived satisfaction with the behavioral component of the program. Among the individuals who provided satisfication with 
Table 2 Change in key outcomes from baseline to 25 weeks

\begin{tabular}{llll}
\hline $\begin{array}{l}\text { Variable } \\
\text { (mean, SD) }\end{array}$ & $\begin{array}{l}\text { Baseline } \\
\text { Mean (SD) }\end{array}$ & $\begin{array}{l}\text { 25-weeks } \\
\text { Mean (SD) }\end{array}$ & Statistic $^{* * *}$ \\
\hline BPRS & $27.00(10.26)$ & $25.06(8.53)$ & $p=.43$ \\
CGI $(n=17)$ & $2.88(1.32)$ & $2.24(1.09)$ & $p=.07$ \\
DAI & $7.89(2.72)$ & $9.83(0.52)$ & $p=.001$ \\
SOFAS & $62.17(18.28)$ & $68.39(15.28)$ & $p=.10$ \\
ESRS-A & $0.06(.24)$ & $0.06(.24)$ & $p=1.00$ \\
Parkinsonism & & & \\
ESRS-A & $0.00(.00)$ & $0.00(.00)$ & $p=$ NA \\
Dystonia & & $0.11(.47)$ & $p=.75$ \\
ESRS-A & $0.17(.51)$ & $0.00(.00)$ & $p=.14$ \\
$\begin{array}{l}\text { Dyskinesia } \\
\text { ESRS-A }\end{array}$ & $0.28(.75)$ & $22.92(5.58)$ & $p=.75$ \\
Akathisia & $22.79(5.02)$ & & \\
BMI & &
\end{tabular}

***: pre/post comparison of baseline to endpoint means for 18 individuals who completed the 25-month trial

BPRS Brief Psychiatric Rating Scale

CGI Clinical Global Impression

$D A /$ Drug Attitude Inventory

SOFAS Social and Occupational Assessmsent of Functioning Scale

ESRS- $A$ Extrapyramidal Symptoms Scale-Abbreviated version

$B M I$ Body Mass Index

intervention input at 25 weeks, $N=18$ (100\%) strongly agreed or agreed that CAE was useful to them.

\section{Discussion}

The purpose of the pilot trial was to determine the feasibility of providing combined treatment with a behavioral program intended to promote medication adherence combined with LAI (haloperidol decanoate) among poorly adherent Tanzanian with CPD. Based on this preliminary experience, the CAE-L intervention appears to be feasible and has high acceptability among patients. As the enrolled sample had minimal psychotic symptoms at baseline, there was minimal symptom improvement. There are several aspects of this pilot work that are worth noting which have implications for clinical care and future research planning.

Our sample appears to have similarities with other reports from SSA. A recent review of treatments for schizophrenia in SSA by Chidarakire and colleagues highlighted the limited mental health services related to financial constraints, lack of qualified mental health professionals, and problems in care access [34]. In this review, the majority of people with schizophrenia were treated with first-generation antipsychotics [35-37]. In our sample, the most common previous antipsychotic drugs were the first-generation antipsychotic drugs haloperidol, chlorpromazine, and promethazine. Individuals in our sample had generally extensive histories of previous relapse. A report by Kazadi et al. found that many patients with CPD discontinue their medication [36]. While our sample was, a priori, confined to individuals known to be poorly adherent, this is a sizable chunk of the CPD population and could be a useful program, especially among individuals who have had recent relapse due to poor adherence. Families in the review by Chidarakire reported a high level of burden associated with caring for a relative with CPD [34]. In our sample, we did not assess family distress, but found that approximately half of individuals with CPD also had a family psychiatric history, a factor that seems likely to add to overall family burden. Future work might include involving family members more extensively as part of patient recovery.

Of the 19 individuals who completed baseline assessments in the study reported here, 18 (95\%) remained on the CAE-L intervention at 25-week follow-up. The LAI also appeared to be relatively well tolerated with only one SAE during the 6-month study period, a hospitalization that was due to substance use relapse and not deemed to be related to study participation. The most common side effects appeared to be related to extrapyramidal symptoms (tremor, muscle pain, restlessness), sedation and blurred vision, all known potential side effect of haloperidol. All side effects experienced by study participants were mild in severity. Previous studies support the notion that switching to LAI among individuals who are already known to readily tolerate oral haloperidol would not entail increased somatic burden or risk [38].

We saw improvement in self-reported medication treatment adherence between study screening and baseline time-points. This is consistent with other reports for this intervention and likely are due to Hawthorne effects in that observing adherence behavior appears to promote improved adherence, at least for the short term [39]. We did not see change in oral medication adherence from study baseline to the 25-week study endpoint. However, LAI adherence was $100 \%$ in this Tanzanian sample. It is also possible that change in self-reported oral medication adherence could have been obscured by the small number of individuals on oral medication at study endpoint. In this Tanzanian sample, individuals were, for the most part, entirely transitioned off of oral medications once LAI was stabilized/maintained. These findings are in contrast to the U.S. studies with the CAE-L intervention where patients continued to receive a variety of oral drugs in addition to LAI $[13,14]$. Given the disproportionate burden of CPD in SSA, the fact that CAE-L participants in Tanzania no longer were on oral medication could have important clinical implications. For example, LAI non-adherence, if and when it occurs, is more readily detected and clinicians and families have the opportunity to intervene in a timely fashion. 
Our study also did not find change in clinical symptoms, or functional status from study baseline to the 25 -week study endpoint. It is possible that the minimal symptom change could have been due to the fact that individuals were already treated and stabilized on oral haloperidol prior to initiating LAI. Studies conducted in the U.S. using the CAE intervention with individuals who are more symptomatic at baseline suggests that global psychopathology and functional status has potential to improve $[12,14]$. In contrast to the lack of change on symptoms and functioning, we saw significant improvement in attitudes towards medication, a finding that has also been seen in U.S. samples that have received the CAE-L intervention [13, 14]. Our study did not capture results of outcomes beyond the 6-month follow-up time point, however a potentially important domain to assess in future work is whether improved attitudes toward CPD medication treatment translates to longer-term adherence promotion and enhanced recovery.

A recently complete systematic literature (SLR) review of the global literature on antipsychotic medication trials in SSA found only 26 studies published from 1994 to 2019 [16]. Studies were from Nigeria $(n=3)$, South Africa $(n=21)$, Malawi $(n=1)$, and there was 1 multicenter study in Nigeria and South Africa. There were no studies from Tanzania. Like the study reported here, the majority of SLR sample participants were male. The primary adverse effects were changes in metabolic outcomes, a finding not seen in our report. The sample in the study reported here had a BMI considered to be within the normal/health range and lower than one typically sees in reports form the U.S. and other high income countries [40]. Also similar to the SLR, we also did not see significant changes in extrapyramidal symptom measures, possibly related to the fact that our study sample was relatively young (< age 39 years).

Despite the promising preliminary feasibility findings, this study has a number of limitations. The small sample size, single-site enrollment, and the fact that poor medication adherence was one of the inclusion criterion may make findings less generalizable to the full spectrum of patients with CPD in Tanzania. Additionally, since we did not collect data on family burden, it is not possible to make any conclusions regarding how CAE-L may impact families and communities. On the other hand, the fact that the study used resources that might be expected to be available in at least some low-resource settings (a firstgeneration LAI, social workers to deliver the behavioral intervention) may increase generalizability and suggest that future studies are warranted given the pervasiveness and corrosive effects of sub-optimal medication adherence among patients with CPD.
Finally, a key benefit to LAIs is the potential to reduce relapse and subsequent hospitalizations. Because our study was an $\mathrm{NIH}$-funded 2-year project, intended to assess the feasibility and preliminary efficacy of the blended LAI and behavioral intervention, individual study participants were followed for a 6-month time-period. During this time period, there was only 1 individual who was hospitalized. The short duration of follow-up and small sample make it difficult to draw conclusions regarding potential overall impact on hospitalizations and our inability to provide information on hospitalization trajectory is a limitation of our report.

\section{Conclusion}

Combining LAI with a customized adherence enhancement behavioral intervention (CAE-L) is an approach that has potential to advance care for people with CPD in resource-limited settings. While this first-ever testing of CAE-L in poorly adherent patients with CPD in Tanzania is promising, future work needs to include a larger sample and a comparison arm to more clearly determine whether this approach can yield additional benefits beyond current clinical practice.

\begin{abstract}
Abbreviations
CAE-L: Customized Adherence Enhancement with Long-acting injectable medication; NIMH: U.S. National Institute of Mental Health; CPD: Chronic Psychotic Disorders; SSA: Sub-Saharan Africa; LAI: Long-Acting Injectable antipsychotic medication; U.S.: United States; CAE: Customized Adherence Enhancement; ROMI: Rating Of Medication Influences; AMSQ: Attitudes toward Mood Stabilizers Questionnaire; MET: Modified Motivational Enhancement Therapy; TRQ: Tablet Routines Questionnaire; OTT: Oral Tolerance Test; IM: Intramuscularly; AUDIT: Alcohol Use Disorders Identification Test; ASSIST: Alcohol, Smoking and Substance Involvement Screening Test; DAl: Drug Attitude Inventory; BPRS: Brief Psychiatric Rating Scale; CGI: Clinical Global Impressions; SOFAS: Social and Occupational Functioning Scale; CBC: Complete Blood Count; HIV: Human Immunodeficiency Virus; EKG: Electrocardiogram; ESRS-A: Extrapyramidal Symptoms Scale-Abbreviated; SPSS: Statistical Package for the Social Sciences; SAE: Serious Adverse Event; BMI: Body Mass Index; SLR: Systematic Literature Review.
\end{abstract}

\section{Acknowledgements}

The authors appreciate the editorial support and assistance of Ms. Celeste Weise.

\footnotetext{
Authors' contributions

JM developed the study design, helped conduct the study, and participated in analysis and manuscript development. SK developed the study design, helped conduct the study, and participated in analysis and manuscript development. CB led the data analysis and participated in manuscript development. IL helped conduct the study and participated in analysis and manuscript development. CM helped conduct the study. KM helped conduct the study. GN participated in analysis and manuscript development. CB helped refine the study and participated in analysis and manuscript development. KAC helped refine the study and participated in analysis and manuscript development. JBL helped refine the study and participated in analysis and manuscript development. MS developed the study design, helped conduct the study, participated in data analysis, and led the manuscript development. All authors have approved the manuscript and the report is a reflection of team consensus.
} 


\section{Funding}

This study was supported by a grant from the National Institute of Mental Health (NIMH) grant NIMH MH1 14700 (PIs Sajatovic \& Mbwambo). The funding body was not involved in study design, data collection and analysis, results interpretation, or manuscript development.

\section{Availability of data and materials}

The data that support the findings of this study are available on request from the corresponding author (MS) to qualified individuals and with appropriate cross-institutional data use agreements. The data are not publicly available due to their containing information that could compromise the privacy of research participants.

\section{Declarations}

\section{Ethics approval and consent to participate}

The authors assert that all procedures contributing to this work comply with the ethical standards of the relevant national and institutional committees on human experimentation and with the Helsinki Declaration of 1975, as revised in 2008. All procedures involving human subjects/patients were approved by the University Hospitals of Cleveland Institutional Review Board (UHCMC IRB), UHCMC IRB number: 05-17-16 and the Muhimbili University of Health and Allied Sciences Office of Research and Publications Document 2017-12-06/ AECNol.X11/87. Informed consent was obtained from all RCT participants prior to their screening and enrollment.

\section{Consent for publication}

Not applicable.

\section{Competing interests}

In the last 3 years, MS has received research grants from Nuromate, Otsuka, Alkermes, International Society for Bipolar Disorders (ISBD), National Institutes of Health (NIH), Centers for Disease Control and Prevention (CDC), PatientCentered Outcomes Research Institute (PCORI). MS has been a consultant to Alkermes, Otsuka, Janssen, Myriad, Health Analytics, Frontline Medical Communications. MS has received royalties from Springer Press, Johns Hopkins University Press, Oxford Press, UpToDate. MS has also received compensation for the preparation of continuing medical education (CME) Activities form Physician's Institute, MCM Education, CMEology, Potomac Center for Medical Education, Global Medical Education, Creative Educational Concepts. The remaining authors declare no conflict of interest.

\section{Author details}

${ }^{1}$ Department of Psychiatry and Mental Health, Muhimbili University of Health and Allied Sciences, School of Medicine, Dar es Salaam, Tanzania. ${ }^{2}$ Frances Payne Bolton School of Nursing, Case Western Reserve University, Cleveland, $\mathrm{OH}$, USA. ${ }^{3}$ Department of Psychiatry and Neurological \& Behavioral Outcomes Center, Case Western Reserve University School of Medicine and University Hospitals Case Medical Center, Cleveland, OH, USA. ${ }^{4}$ Department of Psychiatry \& of Neurology, Case Western Reserve University School of Medicine and University Hospitals Cleveland Medical Center, W.O. Walker Bldg, 7th Floor, 10524 Euclid Avenue, Cleveland, OH 44106, USA.

Received: 14 September 2021 Accepted: 13 December 2021

Published online: 27 January 2022

\section{References}

1. Charlson FJ, Diminic S, Lund C, Degenhardt L, Whiteford HA. Mental and substance use disorders in sub-Saharan Africa: predictions of epidemiological changes and mental health workforce requirements for the next 40 years. PLoS One. 2014;9(10):e110208.

2. Adeponle AB, Thombs BD, Adelekan ML, Kirmayer LJ. Family participation in treatment, post-discharge appointment and medication adherence at a Nigerian psychiatric hospital. Brit J Psychiat. 2009;194(1):86-7.

3. Adewuya AO, Owoeye OA, Erinfolami AR, Coker AO, Ogun OC, Okewole $\mathrm{AO}$, et al. Prevalence and correlates of poor medication adherence amongst psychiatric outpatients in southwestern Nigeria. Gen Hosp Psychiat. 2009;31(2):167-74.
4. Danladi J, Falang KD, Barde RA, Jimam NS, Dangiwa DA, Jimoh HO. Pharmaceutical care and medication adherence in management of psychosis in a Nigerian tertiary hospital. J Res Pharm Pract. 2013;2(2):83-7.

5. Ho Odo OS, Agbonile IO, Esan PO. Assessment of adherence to psychotropic medications among outpatients at the pharmacy department of a psychiatric hospital in Benin City. Nigeria Asian J Pharm. 2014;194(1):86-7.

6. Sariah $\mathrm{AE}$, Outwater $\mathrm{AH}$, Malima KI. Risk and protective factors for relapse among individuals with schizophrenia: a qualitative study in Dar Es Salaam. Tanzania BMC Psychiatry. 2014;14:240.

7. Kafula M, Ugburo E, Kibuule D. Consumption of psychotropic medicines at a referral hospital in Namibia: findings and implications. African Health Sciences. 2020:1000-10.

8. Gilmer TP, Dolder CR, Lacro JP, Folsom DP, Lindamer L, Garcia P, et al. Adherence to treatment with antipsychotic medication and health care costs among medicaid beneficiaries with schizophrenia. Am J Psychiat. 2004;161(4):692-9.

9. Sajatovic M, Dawson NV, Perzynski AT, Blixen CE, Bialko CS, McKibbin CL, et al. Best practices: optimizing care for people with serious mental illness and comorbid diabetes. Psychiatr Serv. 2011;62(9):1001-3.

10. Jenkins JH, Strauss ME, Carpenter EA, Miller D, Floersch J, Sajatovic M. Subjective experience of recovery from schizophrenia-related disorders and atypical antipsychotics. Int J Soc Psychiatry. 2005;51(3):211-27.

11. Mueser KT, Corrigan PW, Hilton DW, Tanzman B, Schaub A, Gingerich S, et al. Illness management and recovery: a review of the research. Psychiatr Serv. 2002;53(10):1272-84.

12. Zygmunt A, Olfson M, Boyer CA, Mechanic D. Interventions to improve medication adherence in schizophrenia. Am J Psychiat. 2002;159(10):1653-64.

13. Collins CM, Aebi ME, Levin JB, Tatsuoka C, Cassidy KA, Sajatovic M. Post-hoc analysis of two clinical trials examining customized adherence enhancement plus long acting injectable antipsychotic (CAE-L) in highrisk individuals with serious mental illness. Schizophr Res. 2018.

14. Sajatovic M, Levin J, Ramirez LF, Hahn DY, Tatsuoka C, Bialko CS, et al. Prospective trial of customized adherence enhancement plus long-acting injectable antipsychotic medication in homeless or recently homeless individuals with schizophrenia or schizoaffective disorder. J Clin Psychiatry. 2013;74(12):1249-55.

15. Mbwambo J, Kaaya S, Lema I, Blixen C, Cassidy KA, Levin J, et al. A customized adherence enhancement program combined with long-acting injectable antipsychotic medication (CAE-L) for poorly adherent patients with chronic psychotic disorder in Tanzania: a pilot study methodological report. Heliyon. 2017:e01763.

16. Kumar S, Sudhakar S, Sajatovic M, Levin JB. Antipsychotic medication in sub-Saharan Africa: a systematic literature review. J Clin Psychopharmacol. 2020;40(6):541-52.

17. Sajatovic M, Levin J, Ramirez F, et al. A concierge model of customized adherence enhancement plus long-active injectable antipsychotic in individuals at risk for treatment non-adherence and for homelessness. In: 55th Annual Meeting of the American College of Neuropsychopharmacology (ACNP). Florida: Hollywood; 2016.

18. Sajatovic M, Levin J, Tatsuoka C, Micula-Gondek W, Williams TD, Bialko CS, et al. Customized adherence enhancement for individuals with bipolar disorder receiving antipsychotic therapy. Psychiatr Serv. 2012;63(2):176-8.

19. Sajatovic M, Levin J, Tatsuoka C, Micula-Gondek W, Fuentes-Casiano E, Bialko CS, et al. Six-month outcomes of customized adherence enhancement (CAE) therapy in bipolar disorder. Bipolar Disord. 2012;14(3):291-300.

20. Weiden P, Rapkin B, Mott T, Zygmunt A, Goldman D, Horvitz-Lennon $M$, et al. Rating of medication influences (ROMI) scale in schizophrenia. Schizophr Bull. 1994;20(2):297-310.

21. Adams J, Scott J. Predicting medication adherence in severe mental disorders. Acta Psychiatr Scand. 2000;101(2):119-24.

22. Harvey NS. The development and descriptive use of the Lithium attitudes questionnaire. J Affect Disord. 1991:22(4):211-9.

23. Velligan DI, Weiden PJ, Sajatovic M, Scott J, Carpenter D, Ross R, et al. Strategies for addressing adherence problems in patients with serious and persistent mental illness: recommendations from the expert consensus guidelines. J Psychiatr Pract. 2010;16(5):306-24.

24. Peet M, Harvey NS. Lithium maintenance: 1. A standard education programme for patients. Br J Psychiatry. 1991;158:197-200. 
25. Scott J, Pope M. Nonadherence with mood stabilizers: prevalence and predictors. J Clin Psychiatry. 2002;63(5):384-90.

26. Chaudhry S, Jin L, Meltzer D. Use of a self-report-generated Charlson comorbidity index for predicting mortality. Med Care. 2005;43(6):607-15.

27. Group WHOAW. The alcohol, smoking and substance involvement screening test (ASSIST): development, reliability, and feasibility. Addiction. 2002;97:1183-94.

28. Saunders JB, Aasland OG, Babor TF, de la Fuente JR, Grant M. Development of the Alcohol Use Disorders Identification Test (AUDIT):WHO Collaborative Project on Early Detection of Persons with Harmful Alcohol Consumption--Il. Addiction. 1993;88(6):791-804.

29. Awad AG. Subjective response to neuroleptics in schizophrenia. Schizophr Bull. 1993;19(3):609-18.

30. Overall JE, Gorham DR. The brief psychiatric rating scale. Psychol Rep. 1962;10:799-812.

31. Guy W. ECDEU assessment manual for psychopharmacology. Rockville, MD: U.S. Department of Health, Education, and Welfare; 1976.

32. Morosini PL, Magliano L, Brambilla L, Ugolini S, Pioli R. Development, reliability and acceptability of a new version of the DSM-IV social and occupational functioning assessment scale (SOFAS) to assess routine social functioning. Acta Psychiatr Scand. 2000;101(4):323-9.

33. Chouinard G, Rosschouinard A, Annable L, Jones BD. Extrapyramidal symptom rating-scale. Can J Neurol Sci. 1980;7(3):233.

34. Chidarikire S, Cross M, Skinner I, Cleary M. Treatments for people living with schizophrenia in sub-Saharan Africa: an adapted realist review. Int Nurs Rev. 2018;65(1):78-92.

35. Esan O. Persistence in treatment for one year among patients in Nigeria with first-episode schizophrenia. Psychiatr Serv. 2014;65(9):1174-6.

36. Kazadi NJB, Moosa MYH, Jeenah FY. Factors associated with relapse in schizophrenia. Sajp-S Afr J Psychi. 2008;14(2):52-+.

37. Van Rensburg AJ, Oloruniu S. Diagnosis and treatment of schizophrenia in a general hospital based acute psychiatric ward. African J Psychiatry. 2010;13(3):204-10.

38. Barbui C, Bertolini F, Bartoli F, Calandra C, Callegari C, Carrà G, et al. Reasons for initiating long-acting antipsychotics in psychiatric practice: findings from the STAR network depot study. Ther Adv Psychopharmacol. 2020;10:2045125320978102.

39. Sajatovic M, Levin J, Ramirez L, Cassidy K, Mcnamara N, Fuentes-Casiano E, et al. Long-acting injectable antipsychotic medication + customized adherence enhancement in poorly adherence patients with bipolar disorder. Prim Care Companion CNS Disord. 2021;23(5):20m0288. https:// doi.org/10.4088/PCC.20m02888

40. Khin NA, Chen YF, Yang Y, Yang P, Laughren TP. Exploratory analyses of efficacy data from schizophrenia trials in support of new drug applications submitted to the US Food and Drug Administration. J Clin Psychiatry. 2012;73(6):856-64.

\section{Publisher's Note}

Springer Nature remains neutral with regard to jurisdictional claims in published maps and institutional affiliations.

Ready to submit your research? Choose BMC and benefit from:

- fast, convenient online submission

- thorough peer review by experienced researchers in your field

- rapid publication on acceptance

- support for research data, including large and complex data types

- gold Open Access which fosters wider collaboration and increased citations

- maximum visibility for your research: over $100 \mathrm{M}$ website views per year

At BMC, research is always in progress.

Learn more biomedcentral.com/submissions 